\title{
Review
}

\section{Live Free or Die}

\section{Tales of Homeless (Cells) in Cancer}

\author{
Craig Horbinski, ${ }^{*}$ Colton Mojesky, ${ }^{\dagger}$ \\ and Natasha Kyprianou*† \\ From the Departments of Patbology, ${ }^{*}$ Surgery/Urology, ${ }^{\dagger}$ \\ and Molecular Biochemistry, ${ }^{\ddagger}$ University of Kentucky, \\ Lexington, Kentucky
}

Anoikis, programed cell death that occurs on cell detachment from the extracellular matrix, thus disrupting integrin-ligand interactions, is a critical mechanism in preventing ectopic cell growth or attachment to an inappropriate matrix. Anoikis prevents shed epithelial cells from colonizing elsewhere and is thus essential for maintaining tissue organization. Lack of integrin ligation leads to decreased focal adhesion kinase and integrin-linked kinase activity, which impairs downstream survival signaling. Consequently, targeting tumor cell survival by triggering anoikis provides a unique molecular basis for novel therapeutic targeting of tumors before initiation of metastasis. The two major cell death pathways involved in anoikis signaling are apoptosis and autophagy; growing evidence suggests an extensive crosstalk between the two killing modes as well as contextdependent cooperation and antagonism. This review discusses the functional integration between the two modes of cell death converging at anoikis, including key molecules of interaction such as Beclin 1 , reactive oxygen species, extracellular signal-related kinase, and death-associated protein kinase. The involvement of other apoptotic effectors such as Bcl-2, p53, and FLICE inhibitory protein in cancer cell anoikis is also discussed. Dissecting the mechanistic players in the cellular response may be of high clinical significance in identifying effective approaches in reversing anoikis resistance in primary tumor cells and, consequently, impairing metastasis. (Am J Pathol 2010, 177:1044-1052; DOI: 10.2353/ajpatb.2010.091270)

Cancer is lethal in large part because of its ability to metastasize to remote locations. Indeed, if metastasis did not exist as a phenomenon, and every solid neoplasm was simply a matter of resection and management of local recurrences, the term "cancer" would lose much of its negative impact on health and would not be the second leading cause of death in the United States. Despite the medical community's most assiduous attempts at early detection before metastatic capability is acquired, many cancers either simply cannot be detected effectively at an early stage (such as ovarian carcinoma) or frequently metastasize at an early stage of development (such as melanoma). Even in cancers amenable to early detection, including carcinomas of the prostate or colon, many patients still develop metastatic disease that ultimately proves to be fatal.

With the notable exception of mature hematopoietic cells, those of the epithelium and mesenchyme depend on cell-cell and cell-matrix interactions for proper differentiation and survival. Anoikis is the term first used to describe the process wherein cells that lose these interactions die-in effect, killing themselves rather than risking killing the organism with tumor spread and ectopic growth. ${ }^{1}$ Thus far, the primary effector mechanisms of anoikis appear to be autophagy and apoptosis. The newness of anoikis and autophagy in cancer research is underscored by the fact that, in PubMed, nearly 70,000 cancer-oriented articles to date have dealt with apoptosis, whereas only about 1000 were concerned with autophagy and barely more than 700 with anoikis. Anoikis, or apoptosis that is inhibited by cell-matrix contact, is regulated by apoptosis-signaling mechanisms. ${ }^{1}$ In certain circumstances, however, suppression of apoptosis is insufficient to abrogate cell death via anoikis, ${ }^{2}$ and autophagy emerges as an execution backup to apoptosis. ${ }^{3}$ This process might not always be to the benefit of the organism, as autophagy is itself capable of either promoting or inhibiting apoptosis, and apoptotic pathways can in turn modulate autophagy, ${ }^{4}$ pushing cells into a survival mode. Thus, there is a great deal of complexity in the functional interplay between apoptosis and autoph-

\footnotetext{
Accepted for publication April 29, 2010.

CME Disclosure: None of the authors disclosed any relevant financia relationships.

Address reprint requests to Dr. Natasha Kyprianou, Ph.D., Department of Surgery, Division of Urology, MS 283, University of Kentucky College of Medicine, 800 Rose St., Lexington, KY 40536. E-mail: nkypr2@uky.edu.
} 
agy signaling pathways in anoikis. For anoikis to be thwarted and cancer cells to proceed in their metastatic journey, tumors must stumble on the right way to both arrest apoptosis and use autophagy in a way that promotes their survival. Understanding the functional crosstalk between apoptosis and autophagy signaling pathways is a prerequisite to development of effective means of impairing metastasis. In this review we aim to summarize the current knowledge of such cross-talk, which first necessitates an introduction to each process as currently understood.

\section{Programming Cell Death Pathways}

\section{Apoptosis: Cellular Suicide}

Apoptosis is the archetype of programed cell death and is divisible into two pathways: extrinsic (death receptor-dependent) and intrinsic (mitochondria-dependent). ${ }^{5}$ Both pathways ultimately cause activation of cysteine proteases, called caspases, which enzymatically destroy the cell in a manner that, unlike necrosis, avoids inflammation. The extrinsic (also called the death receptor) pathway is initiated when membrane-bound death receptors such as Fas receptor, tumor necrosis factor (TNF) receptor 1 (TNFR1), and TNF-related apoptosis-inducing ligand receptor-1 or -2 (TRAIL R1/R2), all of which are part of the TNF receptor superfamily, bind with soluble ligands such as Fas ligand, TNF- $\alpha$, or TRAIL, respectively. When Fas is stimulated, the Fas-associated death domain (FADD) adapter molecule directly triggers procaspase-8 oligomerization and autocleavage into active caspase-8 monomers. ${ }^{6}$ This causes cytochrome $c$ release from the mitochondria and activation of caspase-3, -7 , and -9 , ultimately resulting in proteolytic cleavage, nuclear fragmentation, and cellular breakup. Of note, FADD promotion of procaspase-8 oligomerization can be blocked by FLICE inhibitory protein (FLIP). ${ }^{7}$

When TNFR1 is activated, one of two complexes forms. Complex I consists of tumor necrosis factor receptor-1associated death domain (TRADD), cellular inhibitor of apoptosis-1 (CIAP1), receptor-interacting protein (RIP)-1 kinase, and TNF receptor-associated factor-2 (TRAF2). This complex results in nuclear factor $-\kappa \mathrm{B}$ activation and survival. However, when TRADD dissociates from TNFR1, it can recruit FADD and procaspase-8 to TNFR1, forming complex II. This second complex results in a cascade similar to that seen with FADD. Whether the effects of complex I or II predominate seems to depend on whether nuclear factor $-\kappa B$ activation is sufficient to promote FLIPmediated caspase- 8 suppression. ${ }^{8}$

The intrinsic (also called the mitochondrial) pathway, on the other hand, focuses on the mitochondria and generally occurs in response to cell stressors including oxidative stress, DNA damage, viral infection, and UV radiation. This pathway is intracellularly mediated by the $\mathrm{Bcl}-2$ family of proteins (Figure 1). The common effect of these extrinsic factors is to increase mitochondrial membrane permeability, in turn facilitating cytochrome $c$ release. The presence of cytosolic cytochrome $c$ triggers the formation of a complex consisting of seven inhibitor of apoptosis proteins (Apaf)- 1 and seven caspase-9 monomers known as the apoptosome. ${ }^{9}$ This apoptosome formation causes caspase- 9 activation, leading to cell execution via apoptosis. Key regulators of cytochrome $c$ release include the $\mathrm{Bcl}-2$ family of proteins, each of which has either pro- or anti-apoptotic effects. Some of the potent apoptosis suppressors, such as Bcl-2 or Bcl-XL, localize to the mitochondrial outer membrane and prevent cytochrome $c$ release, thereby suppressing apopto-

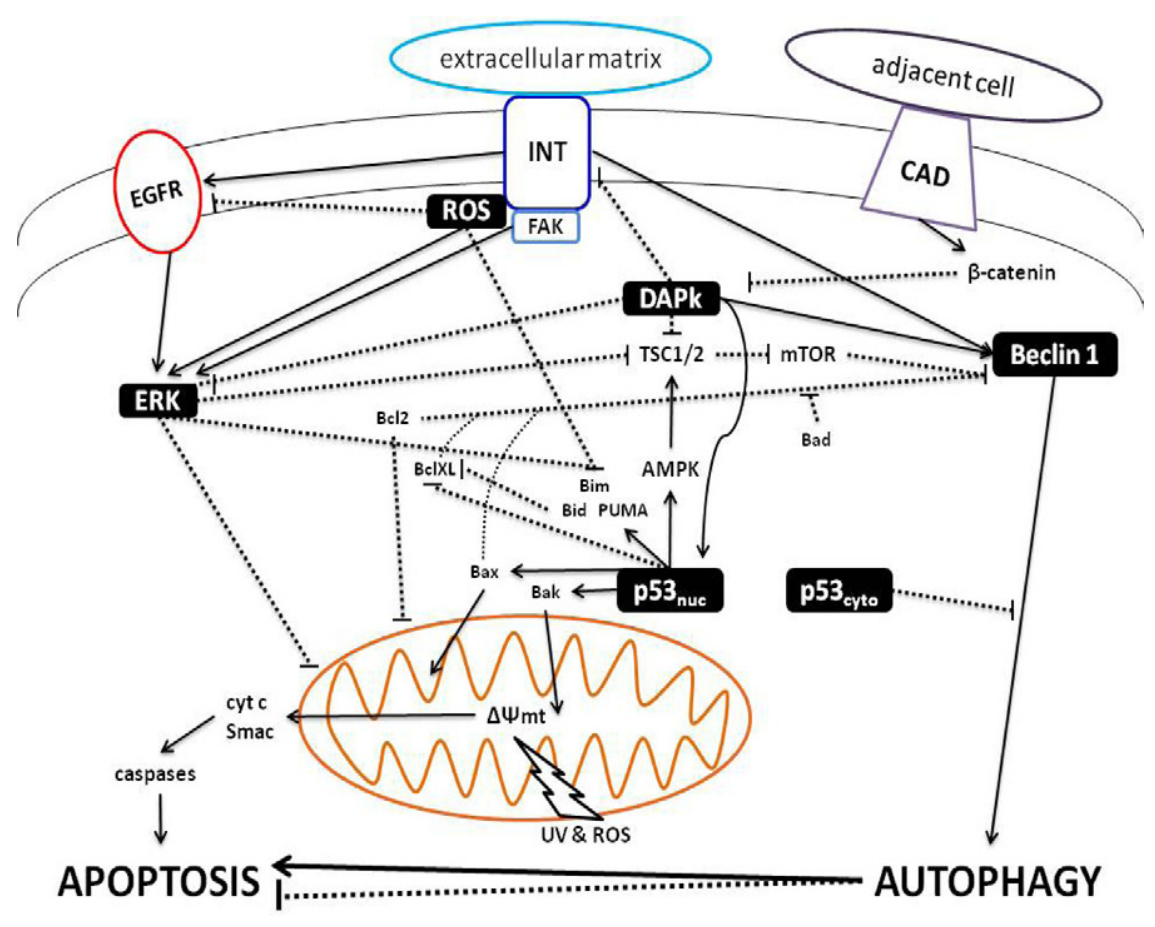

Figure 1. Cross-talks of death: encounters between autophagy and apoptosis converge in anoikis. Key points of functional exchange and signaling interactions engage integrin-stimulated ROS, DAPk, Beclin 1, and ERK. The activity of BCL-2 family members is multifaceted. The family consists of the antiapoptotic group, including the major apoptosis suppressor $\mathrm{Bcl}-2$; the proapoptotic "multi-BH domain" group, including Bax and Bak; and the proapoptotic "BH3-only" group, including Bid. It is the ratio of pro- and antiapoptotic proteins that controls the cellular vulnerability to apoptosis. $\mathrm{Bcl}-2$ helps maintain both mitochondrial membrane homeostasis and calcium homeostasis within the cell. Bax and Bak can respond to an intrinsic death stimulus by translocating (Bax), oligomerizing and inserting into the outer mitochondrial membrane to promote permeabilization. Proteolytic Bid cleavage mediated by caspase- 8 leads to Bid myristoylation, mitochondrial localization, and MOMP induction, an event that suggests a cross-talk event between the two major apoptotic pathways. E-cadherin regulates the desmosomal junctions and its loss confers resistance to anoikis. All players act as transducers between cell-cell and cell-extracellular matrix signals to promote autophagy and/or apoptosis, either resulting in the death of the detached cell or enhanced survival toward metastatic spread. INT, integrins; cyt c, cytochrome $c ; \Delta \psi \mathrm{mt}$, mitochondrial membrane potential. 
sis. Such activity is directly inhibited by tumor protein 53 (p53). ${ }^{10}$ In contrast, BCL-2 homology region (BH) 3-only pro-apoptotic proteins such as Bid, Bim, and PUMA bind to and inhibit $\mathrm{Bcl}-2$ and $\mathrm{Bcl}-\mathrm{XL},{ }^{11}$ thus promoting apoptosis, as illustrated in Figure 1. When the net activity of all these proteins is in favor of death, Bax and Bak work in unison to increase mitochondrial membrane permeability, leading to cytochrome $c$ release, which in turn activates the aforementioned caspase cascade and apoptosis. ${ }^{12,13}$ Additional proteins released from the mitochondria can also trigger apoptosis, either through inactivation of inhibitors of apoptosis proteins and/or through the release of second mitochondria-derived activator of caspases (Smac/ DIABLO), which promotes caspase activation through inhibition of CIAP1. Signaling cross-talk between the intrinsic and extrinsic pathways seems to include Smac/ DIABLO-mediated potentiation of death receptor signaling and caspase-8-mediated activation of Bid. ${ }^{5}$

\section{Autophagy: Cells Starving to Death}

Autophagy is triggered by nutrient deprivation, starvation, and other stress stimuli, on which the cell breaks down and recycles vital internal components. ${ }^{5}$ Unlike the ubiquitinproteasome system, which degrades short-lived proteins, macroautophagy (also called mitophagy; hereafter referred to simply as autophagy) processes long-standing macromolecules and whole organelles, most notably the mitochondrion. ${ }^{14,15}$ This highly conserved process occurs in a wide range of biological phenomena, including development, starvation, immunity, aging, neurodegeneration, and cancer. Conceptually, autophagy is divided into four phases. The first phase, called induction, occurs in response to environmental stressors such as nutrient deprivation, oxidative stress, infection, or hypoxia. This leads to the second phase, wherein structures targeted for degradation are enveloped by a membrane called an autophagophore. The resulting double-layered membrane is an autophagosome. Third, this autophagosome fuses with a lysosome containing hydrolytic enzymes, forming an autophagolysosome. The fourth and final phase consists of the actual enzymatic degradation and recycling of materials. ${ }^{14}$ Thus, the intracellular digestion of macromolecules during the autophagic process is a self-conserved response.

The dynamics of occurrence of the autophagic phenomenon, although not completely understood, are obviously highly sophisticated and tightly regulated, involving a number of complex-forming proteins. ${ }^{5,16}$ Under proautophagic conditions such as amino acid deprivation, Beclin 1 (Atg6 in yeast cells), phosphatidylinositol 3-kinase, and Vps34 aggregate and promote induction. A group of autophagy (Atg) proteins, including Atg12, Atg5, and Atg16, form a complex via Atg7 and Atg10, the latter two proteins acting in a manner analogous to ubiquitin-like enzymes E1 and E2, respectively. Next, microtubule-associated protein 1 light chain 3 (LC3/Atg8) is activated by Atg 4 and Atg7. LC3 is then ligated to phosphatidylethanolamine via Atg3. The Atg12-Atg5-Atg16 complex, in conjunction with LC3-phosphatidylethanol- amine, facilitates autophagophore membrane elongation and autophagosome formation. A key regulator of autophagy is the mammalian target of rapamycin (mTOR) which, on activation, becomes a potent inhibitor of Atg12Atg5-Atg16 complex formation. ${ }^{17}$

\section{Anoikis: Homelessness Finds Death}

Anoikis, meaning "homeless" in Greek, occurs when cellular contact with the proper type of extracellular matrix $(\mathrm{ECM})$ is lost. ${ }^{1,18}$ For tumor cells to become metastatic, they must survive detachment from the ECM and resist the apoptotic stimuli associated with invasion, migration, and metastasis by activating survival mechanisms. The molecular events contributing to tumor cell progression to metastasis are mediated by integrins, which, on engagement with components of the ECM, reorganize to form adhesion complexes. In the context of solid tumors, such changes occur during tissue invasion, detachment into the vascular lymphatic space, and/or implantation into a foreign site with unfamiliar ECM. Any of these events can cause disruption of the $\alpha$ and $\beta$ subunits of integrin heterodimer receptors, resulting in the deactivation of focal adhesion kinase (FAK) and Src family kinases, as well as integrin-linked kinase. In turn, this leads to attenuation of pro-survival pathways including mitogen-activated protein kinase/extracellular signal-related kinase (ERK), Akt/phosphatase and tensin homolog (PTEN), and nuclear factor $-\kappa \mathrm{B}$. Freed from prior inhibition, proapoptotic proteins such as Bax and Bak then destabilize the mitochondrial membrane potential and increase cytochrome $c$ release, activating the caspase pathway and triggering intrinsic apoptosis. Alternatively (or additionally), integrin disruption can result in increased expression of Fas ligand-binding cell surface receptors, in turn promoting FADD-mediated caspase activation and extrinsic apoptosis. Compelling evidence indicates that loss of E-cadherin (a major desmosomal junction coordinator) in breast tumor cells in vitro, as well as in an in vivo model of mammary tumorigenesis, confers anoikis resistance and increased angiogenesis, thus contributing to metastasis. ${ }^{19,20}$ What has become increasingly apparent from the in vivo evidence is that the state of "homelessness" is not exclusive to tumor epithelial cells; for example, during angiogenesis, endothelial cells are in a dynamically fluid state and lack firm attachment to the ECM, thus becoming vulnerable to anoikis.

Resistance to anoikis can be conferred by diverse molecular mechanisms, including constitutive activation of FAK, ${ }^{21}$ epidermal growth factor receptor (EGFR)-mediated Src activation, ${ }^{22}$ increased cytosolic FLIP, ${ }^{23}$ and overexpression of $\beta$-catenin. ${ }^{24}$ In human colorectal cancer cells the DR5 (TRAIL death receptor) mediates death signals for anoikis via the extrinsic receptor pathway. ${ }^{25}$ One molecule of particular interest in this extrinsic death signaling is FLIP, the primary endogenous inhibitor of the death receptor pathway. FLIP consists of two death effector domains and a $\mathrm{C}$ terminus caspase-like domain that is structurally similar to caspase-8. On binding, FLIP substitutes an active-site cysteine residue to tyrosine, 
leading to inactivation of caspase-8. FLIP has higher affinity to the death-inducing signaling complex compared with caspase-8, thus blocking caspase-8 recruitment and subsequent activation. ${ }^{26}$ In normal cells, on loss of cell contact with ECM, Fas ligands and Fas receptor are up-regulated, whereas FLIP expression is down-regulated. These changes trigger downstream caspase-8 activation in a FADD-dependent manner, leading to apoptosis. ${ }^{27}$ In malignant cells, even after they lose contact with the ECM, caspase-8 is not activated despite increased expression of FAS ligands and FAS receptor. This may occur because of the failure of cancer cells to down-regulate FLIP after detachment. ${ }^{28}$ Targeting FLIP in anoikis-resistant cells may therefore inhibit metastatic disease. This is a promising avenue of research, because chemical inhibition of FLIP sensitizes cells to apoptosis and restores anoikis in malignant cells, yet does not induce apoptosis in normal adherent cells. ${ }^{29}$ A functional link between FAK and death receptors has been established via the ability of FAK to bind to the death domain kinase RIP. ${ }^{30}$ These studies enabled a new mechanistic insight into FAK survival signaling mediated by binding to RIP and inhibiting its interaction with the death receptor complex. Moreover, recent evidence indicates a critical role for RIP in mediating anoikis by being shuttled between CD95/Fas-mediated death and FAK/integrin survival signaling. ${ }^{31}$ The dynamic of this functional interaction/exchange is robust.

A controversial component linked to anoikis resistance and tumor progression is the epithelial to mesenchymal transition, wherein tumor cells acquire spindly morphology and have reduced cell-cell interactions and increased migratory ability. ${ }^{32}$ The cytoskeletal rearrangements that tumor cells undergo during the epithelial to mesenchymal transition and during migration and invasion of blood vessels ${ }^{33}$ may determine the plasticity of the tumor cells and their sensitivity to anoikis. But is this a mere reflection of cellular changes adjusting to the tumor microenvironment or spatial relocation of interactive epithelial and endothelial cells with the ECM? Pathological epithelial to mesenchymal transition in tumor cells results from transcriptional reprogramming of abnormal survival signals via an array of growth factor receptor signaling, including the transforming growth factor- $\beta$ receptor pathway, and survival kinases such as Akt and phosphatidylinositol 3-kinase, all potent regulators of the epithelial to mesenchymal transition and promoters of metastasis. ${ }^{32}$ Another recently identified parameter is intratumoral hypoxia, a phenomenon that triggers hypoxia-inducible factor- $1 \alpha$ expression in the tumor cells, in turn promoting anoikis resistance through integrin inhibition. ${ }^{34}$

Numerous efforts have been made to develop models of anoikis, specifically elucidating the mechanisms by which autophagy and apoptosis interact to dictate the outcomes of anoikis in an in vivo setting of tumorigenesis. The following sections discuss one such model, some of the key players shown to participate in cross-talk, and the molecular sophistication with which apoptosis and autophagy are actively engaged in anoikis.

\section{In Vitro Modeling of Anoikis: Wear Your Three-Dimensional Glasses}

Elegant studies using three-dimensional mammary gland acini as in vitro models for studying the mechanisms of anoikis have provided new insights into the phenomenon. ${ }^{2,3}$ Normally, all glandular acini maintain an open lumen by pruning back epithelial cells that mistakenly overgrow and attempt to fill in the luminal space. Such pruning occurs because epithelial cells need attachment to an underlying basement membrane, otherwise cell-toECM interactions are disrupted and a variety of the aforementioned pathways that lead to apoptosis are triggered. In vitro three-dimensional cultures readily show the differences between normal and neoplastic mammary cells, as the former will develop acinar-like structures whereas the latter show disorganized growth and luminal filling. ${ }^{35}$ Their use has led, for example, to the discovery of novel roles for E-cadherin in differentiation and growth arrest via PTEN. ${ }^{36}$

MCF-10A mammary cells deprived of ECM contact undergo anoikis and autophagy. When autophagy is blocked by knocking down Atgs, apoptosis is induced. Furthermore, cells overexpressing $\mathrm{Bcl}-2$ can survive on detachment from the ECM, but only if autophagy is allowed to proceed. Thus, autophagy can thwart anoikis whether or not the apoptotic machinery is functional. Mechanistically, this occurs via persistent activation of $5^{\prime}$-adenosine monophosphate-activated protein kinase (AMPK) and eukaryotic initiation factor- $2 \alpha$, the former being a known inhibitor of mTOR via tuberous sclerosis complex (TSC) 2 activation. ${ }^{37}$ In contrast, if MCF-10A cells attempt to fill the luminal space (analogous to breast carcinoma in situ), separation from the ECM is inevitable. During luminal filling, even when apoptosis is blocked, TRAIL can still promote cell death and luminal clearing via autophagy activation. ${ }^{38}$ Such apparent contradictory roles for autophagy in either promoting cell death or survival suggest that, unlike apoptosis, which always results in cell death, autophagy during anoikis is characterized by multilayered complexity and can produce widely different results, depending on its trigger and timing of induction. ${ }^{39}$

\section{Cell Death Players Cross-Talk in Anoikis}

\section{Beclin 1: Functional Exchange Coordinator}

Beclin 1 is a key point where cross-talk between apoptosis and autophagy has been described. This protein, known as Atg6 in yeast cells, is an early component of the autophagic vesicle that is destined to later become an autophagosome (Figure 1). Beclin 1 contains a $\mathrm{BH} 3$ domain capable of binding to the anti-apoptotic Bcl-2/ $\mathrm{Bcl}-\mathrm{xL}$ proteins. ${ }^{40}$ When this happens, Atg6/Beclin 1 activity and subsequent autophagosome formation can be blocked. ${ }^{41}$ On the other hand, the pro-apoptotic BH3only domain protein Bad directly removes this inhibition, thereby facilitating both autophagy and apoptosis. ${ }^{42}$ Adding to the complexity of these interactions is the 
recent finding that $\mathrm{Bax}$, a pro-apoptotic $\mathrm{BH} 1-3$ protein, inhibits autophagy by promoting caspase-dependent cleavage of Beclin 1; this interaction is itself completely prevented by $\mathrm{Bcl}-\mathrm{XL} \mathrm{C}^{43}$ Intriguingly, autophagy stimulated by ECM detachment is not sensitive to $\mathrm{Bcl}-2,{ }^{37}$ suggesting that in anoikis, sensitivity to such cross-talk varies. The subcellular distribution/localization of $\mathrm{Bcl}-2$ is also a critical determining factor, as it blocks autophagy when localized to the endoplasmic reticulum and blocks apoptosis when associated with the mitochondria. ${ }^{41}$ Thus, whether a detached cell undergoes autophagy or not depends on its relative pro- or anti-apoptosis status, as well as the context in which autophagy is triggered.

\section{Reactive Oxygen Species: Metabolic Impact on Cell Death}

Reactive oxygen species (ROS) are more than just accidental by-products of cellular metabolism, as they have been shown to participate in cell signaling pathways ranging from morphogenesis to proliferation. The precise way in which ROS are regulated during anoikis and whether they contribute to cell death or survival are controversial topics. In some models, cellular detachment has been shown to result in elevated ROS, in turn contributing to cell death. ${ }^{44}$ In MCF-10A cells with Bim knocked out, separation from the ECM results in elevated ROS and oxidized lipids. ${ }^{2}$ However, when ECV304 cells are attached to ECM, integrin stimulation actually leads to Rac-1-dependent production of ROS, in turn activating Src and ligand-independent activation of the EGFR/ERK pathway plus degradation of Bim, thereby promoting survival. ${ }^{45}$ This anti-anoikis effect can be reversed with antioxidants. ${ }^{46}$ As shown in Figure 1, in primary prostate cells, ROS have been shown to inhibit integrin-induced EGFR signaling and promote cell death. But in those same cells, integrins can also stimulate autophagy, which then prevents caspase activation and apoptosis. ${ }^{47}$

In addition, it is noteworthy that cellular detachment from the ECM has recently been shown to cause ROS production, reduced glucose uptake, and ATP depletion. This process can be rescued via receptor tyrosine kinase promotion of the pentose phosphate pathway, which produces NADPH-reducing equivalents. Exogenous treatment with antioxidants thwarts anoikis in these cells, suggesting that anoikis-mediated cell death depends on elevated ROS. ${ }^{48}$ The implications are significant in the context that, although antioxidants may be helpful in preventing cancer, once cancer status is achieved they may increase resistance to anoikis and consequently the likelihood of metastatic spread. Yet as discussed above, one cannot dismiss the evidence that antioxidant treatment has in some cases restored anoikis sensitivity. ${ }^{46}$ Clearly, further research will help elucidate the precise role(s) that ROS plays in anoikis-mediated apoptosis and autophagy, as well as whether or not antioxidant therapy is a wise idea in patients with preexisting carcinomas.

\section{ERK: Cross-Talk Propagated}

ERK is a serine/threonine protein kinase that participates in a variety of complex signal transduction pathways, including cell survival and proliferation. In response to ROS, activated ERK $1 / 2$ can specifically target the mitochondria, promoting ATP synthase activity, maintaining mitochondrial membrane potential, reducing cytochrome $c$ release, and inactivating Bad. On loss of integrin signaling in normal cells, EGFR levels decrease, ERK is inactivated, Bim increases, and apoptosis occurs. ${ }^{49}$ Moreover, treatment of human stem cells with basic fibroblast growth factor leads to inhibition of Bim and caspase-mediated anoikis via Akt and ERK ${ }^{50}$ Colon carcinoma cells, in contrast, show resistance to anoikis in part through persistent activation of ERK. ${ }^{22}$ In squamous cell carcinomas, nonneoplastic endothelial cells not only supply the tumor with blood but also can secrete interleukin-6, CXCL8, and epidermal growth factor, which stimulates STAT3, Akt, and ERK in the tumor cells. The result is that such endothelial-derived stimuli promote increased migration and reduced anoikis. ${ }^{51}$ However, whereas most studies show that ERK is prosurvival, there is controversial evidence to suggest that this only holds if ERK activation is persistently strong. If ERK is only moderately and intermittently activated, it can exert a proapoptotic effect during anoikis. ${ }^{52}$

Regarding autophagy, both in neoplasms and in response to phorbol esters, ERK can inactivate TSC1/ TSC2, in turn removing inhibition of mTOR. ${ }^{53,54}$ Interestingly, the activated mitogen-activated protein kinase kinase/ERK complex has recently been shown to inhibit mTOR via TSC, thus enhancing Beclin 1-mediated autophagy. Under moderate levels of Beclin 1 expression, such autophagy is cytoprotective, but high Beclin 1 expression and activation cause cell death. ${ }^{55}$ Activated ERK $1 / 2$ also seems to trigger autophagy of damaged mitochondria and may even exert signaling effects from within the organelle. ${ }^{56}$ As with apoptosis, the net effect of ERK in autophagy depends on activity and temporal variations, as only sustained ERK activation in response to 6-hydroxydopamine in Parkinson's disease models causes ERK translocation to the mitochondria, increased autophagy (specifically mitophagy), and cell death rather than cell survival. ${ }^{57,58}$ The emerging scenario is intriguing: depending on its level and temporal timing of activation, ERK can either promote or antagonize autophagy and/or apoptosis, probably serving as a pro-anokis versus anti-anoikis switch (Figure 1).

\section{p53: A Link between Genome Surveillance and Anoikis?}

Given the major impact p53 has in cancer, it is somewhat surprising that the "guardian of the genome" seems to have only a limited role in the apoptosis-autophagy interplay during anoikis. The well known path of apoptosis led by p53 proceeds via up-regulation of pro-apoptotic proteins, including Bax, PUMA, and NOXA, as well as inhibition of $\mathrm{Bcl}-2 .{ }^{4}$ Although the precise role of p53 in 
anoikis has yet to be fully defined, there is emerging evidence implicating its potential involvement in this death phenomenon (Figure 1). Recent elegant studies have mechanistically linked SIK1 in p53-dependent anoikis and metastasis suppression, ${ }^{59}$ whereas earlier reports suggested that suppression of p53 activity contributes to anoikis resistance in squamous cell carcinoma via recruitment of the integrin survival pathway. ${ }^{60}$ Yet as for ERK, the function of p53 in autophagy is far more complex, not only at the expression level but also via intracellular localization. For example, in response to DNA damage, activated p53 translocates to the nucleus and activates the TSC1/TSC2 complex through AMPK, ultimately inhibiting mTOR and promoting autophagy. ${ }^{61}$ In contrast, cytoplasmic p53 blocks the low-level form of autophagy that allows cells to produce ATP and resist energy deprivation. ${ }^{62}$ Neoplastic cells therefore benefit from p53 suppression by resistance to apoptosis and energy/nutrient deprivation, in turn preventing anoikis and facilitating metastasis (Figure 1).

\section{Death-Associated Protein Kinase: Dynamic Death Performance}

Death-associated protein kinase (DAPk) is a serine-threonine cytoskeleton-associated kinase that sensitizes the cell to a variety of apoptosis-inducing signals, including

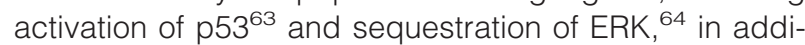
tion to blocking integrin/FAK-dependent cell survival. ${ }^{65}$ This protein also promotes lethal autophagy in response to cellular stresses, ${ }^{66,67}$ at least in part by directly phosphorylating Beclin 1, freeing it from Bcl-xL. ${ }^{68,69}$ Recent work, however, showed that DAPk binds to and disrupts the TSC1/TSC2 complex, resulting in mTOR activation and reduction in autophagy on exposure of HEK293 cells to certain growth factors. ${ }^{70}$ Of interest, colon carcinoma cells can up-regulate $\beta$-catenin after detachment from the ECM, in turn down-regulating DAPk. ${ }^{71}$ Thus, although DAPk promotes anoikis through apoptosis and lethal autophagy, it may also reduce autophagy when the cell is in a progrowth environment.

\section{Autophagy and Apoptosis Converge in Anoikis: Death to the Homeless!}

Apoptosis has only one outcome-the orderly elimination of the cell via activation of its suicidal molecular programs. In contrast, autophagy is much more diverse in its effects, all depending on the target being degraded and the extent of autophagy that is allowed to occur. As discussed above, even if apoptosis is blocked in anoikis, autophagy can either act as a backup for cell death or promote survival. ${ }^{3,36}$ For instance, although autophagy can facilitate apoptosis by generating enough ATP to fuel membrane blebbing, ${ }^{67}$ it may also prevent apoptosis by destroying damaged mitochondria, thereby limiting ROS exposure and cytochrome $c$ release. ${ }^{72,73}$ Impaired autophagy could theoretically promote oncogenesis by allowing accumulation of mutation-inducing by-products, inso- far as Beclin 1 haploinsufficiency has been identified in many carcinomas and transgenic mice lacking one copy of Beclin 1 show neoplastic diathesis. ${ }^{74-76}$ More recent work has shown that inhibition of Beclin 1 and autophagy causes accumulation of p62, elevated ROS, and increased chromosomal instability, resulting in oncogenesis. ${ }^{7-79}$ Ras signaling can also exert proneoplastic effects through down-regulation of Beclin $1 .{ }^{80}$ And, as discussed above, autophagy blocks apoptosis in both adherent prostate cells ${ }^{50}$ and detached mammary epithelial cells. ${ }^{40}$

The interplay platform is richly populated by the primers of death, and detailed dissection of their role has potentially important consequences regarding the development of novel antitumor therapies with the aim of restoring anoikis and inhibiting metastasis. For example, autophagy antagonizes the pro-apoptotic effects of melanoma differentiation-associated gene-7/interleukin-24 gene therapy in leukemic cells in vitro and in vivo. ${ }^{81}$ In colon cancer cells, inhibiting autophagy with chloroquine allows the histone deacetylase inhibitor vorinostat to induce apoptosis, ${ }^{81}$ whereas trifluorothymidine appears to trigger apoptosis more effectively than 5 -fluorouracil because it elicits less autophagy. ${ }^{82}$ Glioma stem-like, CD133-positive cells are more resistant to radiation-induced apoptosis, at least in part because of increased autophagy. ${ }^{83}$ Yet in the context of hypoxia, Beclin 1-mediated autophagy is substantially reduced in neoplastic intestinal cells inherently resistant to hypoxia-induced apoptosis, indicating that autophagy is necessary for apoptosis to occur. ${ }^{84} \mathrm{~A}$ similar pattern of functional interdependency is evident among various tumor cell types: hepatocellular carcinoma cells require autophagy for transforming growth factor- $\beta$-stimulated apoptosis, ${ }^{85}$ whereas up-regulation of autophagy in cervical cancer cells sensitizes them to carboplatin. ${ }^{86}$

These are only a few of the many situations documenting the dynamic and often contradictory interplay that exists between autophagy and apoptosis in dictating cell death. ${ }^{4}$ Resistance to death via anoikis dictates tumor cell survival and provides a molecular basis for therapeutic targeting of metastatic cancer. Indeed, our recent work identified the function of yet another focal adhesion complex protagonist, talin, in conferring anoikis resistance during prostate cancer progression to metastasis. ${ }^{87}$ One cannot, however, consider autophagy as being solely pro- or antisurvival, including in the context of anoikis. Beclin 1, ROS, ERK, and DAPk are key molecules responsible for integrating autophagy and apoptosis, whose effects can differ greatly from one situation to another, rendering therapeutic targeting a challenge (Figure 1). Further dissection of critical anoikis signaling events will enable the induction of anoikis and inhibition of cell survival during the state of homelessness, thus effectively impairing invasion and metastasis. However, there is a cautionary caveat: the exchanges between the two main mechanisms of anoikis-related cell death, apoptosis and autophagy, must be considered in novel regimens purporting to restore anoikis. 


\section{Summary and Future Perspectives}

The knowledge gathered so far from molecular dissection of apoptosis and autophagy signaling allows for combination targeting of the converging points in apoptosis, autophagy, and anoikis death modes. The ultimate goal is suppression not only of primary tumor growth but also of metastatic spread. Recent efforts to dissect the complex anoikis signaling network has already provided valuable insights, facilitating not only its acceptance as a biologically meaningful cell death process but also exploitation of its therapeutic value during cancer initiation and metastatic progression. The cross-talk between anoikis and apoptosis converges at a key activation point of CD95/Fas as it connects RIP, a kinase that shuttles between CD95/Fas-mediated death and FAK-mediated survival pathways.

The current clinical interest surrounding the efficacy of systematically administered short hairpin RNA as an effective therapeutic strategy in human cancers can be applied to anoikis regulators toward impairment of metastatic disease. The success of this approach depends on the ability to discern the targeted proteins that "feed into" the intersection where anoikis meets the other two death operators and to successfully and selectively deliver such short hairpin RNAs to tumor cells. Temporal targeting of anoikis signaling pathways should be precisely programed during the transition from the detachment from the ECM and initiation of the metastatic process. Of immense value would be the implementation of proteomics technology to define a predictive marker or a molecular signature, in the context of the tumor microenvironment, before the onset of metastasis, to enable the selection of cancer patients likely to exhibit therapeutic benefit in response to anoikis resistance "reversal" agents. Free to die or enslaved to their native ECM, homeless cells offer the ultimate sacrifice to stop the spread of cancer.

\section{References}

1. Frisch SM, Francis H: Disruption of epithelial cell-matrix interactions induces apoptosis. J Cell Biol 1994, 124:619-626

2. Mailleux AA, Overholtzer M, Schmelzle T, Bouillet P, Strasser A, Brugge JS: BIM regulates apoptosis during mammary ductal morphogenesis, and its absence reveals alternative cell death mechanisms. Dev Cell 2007, 12:221-234

3. Debnath J, Mills KR, Collins NL, Reginato MJ, Muthuswamy SK, Brugge JS: The role of apoptosis in creating and maintaining luminal space within normal and oncogene-expressing mammary acini. Cell 2002, 111:29-40

4. Eisenberg-Lerner A, Bialik S, Simon HU, Kimchi A: Life and death partners: apoptosis, autophagy and the cross-talk between them. Cell Death Differ 2009, 16:966-975

5. Hotchkiss RS, Strasser A, McDunn JE, Swanson PE: Cell death. N Engl J Med 2009, 361:1570-1583

6. Valmiki MG, Ramos JW: Death effector domain-containing proteins. Cell Mol Life Sci 2009, 66:814-830

7. Thome M, Schneider P, Hofmann K, Fickenscher H, Meinl E, Neipel F, Mattmann C, Burns K, Bodmer JL, Schroter M, Scaffidi C, Krammer PH, Peter ME, Tschopp J: Viral FLICE-inhibitory proteins (FLIPS) prevent apoptosis induced by death receptors. Nature 1997, 386:517-521

8. Micheau O, Tschopp J: Induction of TNF receptor I-mediated apoptosis via two sequential signaling complexes. Cell 2003, 114:181-190
9. Hill MM, Adrain C, Martin SJ: Portrait of a killer: the mitochondrial apoptosome emerges from the shadows. Mol Interv 2003, 3:19-26

10. Tomita Y, Marchenko N, Erster S, Nemajerova A, Dehner A, Klein C, Pan H, Kessler H, Pancoska P, Moll UM: WT p53, but not tumor-derived mutants, bind to $\mathrm{Bcl} 2$ via the DNA binding domain and induce mitochondrial permeabilization. J Biol Chem 2006, 281:8600-8606

11. Yu J, Zhang L, Hwang PM, Kinzler KW, Vogelstein B: PUMA induces the rapid apoptosis of colorectal cancer cells. Mol Cell 2001 7:673-682

12. Wei MC, Zong WX, Cheng EH, Lindsten T, Panoutsakopoulou V, Ross AJ, Roth KA, MacGregor GR, Thompson CB, Korsmeyer SJ: Proapoptotic BAX and BAK: a requisite gateway to mitochondrial dysfunction and death. Science 2001, 292:727-730

13. Zong WX, Lindsten T, Ross AJ, MacGregor GR, Thompson CB: $\mathrm{BH} 3-$ only proteins that bind pro-survival $\mathrm{Bcl}-2$ family members fail to induce apoptosis in the absence of Bax and Bak. Genes Dev 2001, 15:1481-1486

14. Apel A, Zentgraf H, Buchler MW, Herr I: Autophagy-A double-edged sword in oncology. Int J Cancer 2009, 125:991-995

15. Levine B, Klionsky DJ: Development by self-digestion: molecular mechanisms and biological functions of autophagy. Dev Cell 2004, 6:463-477

16. Mizushima N: The pleiotropic role of autophagy: from protein metabolism to bactericide. Cell Death Differ 2005, 12(Suppl 2):1535-1541

17. Pattingre S, Espert L, Biard-Piechaczyk M, Codogno P: Regulation of macroautophagy by mTOR and Beclin 1 complexes. Biochimie 2008 , 90:313-323

18. Chiarugi $P$, Giannoni E: Anoikis: a necessary death program for anchorage-dependent cells. Biochem Pharmacol 2008, 76:1352-1364

19. Onder TT, Gupta PB, Mani SA, Yang J, Lander, ES, and Weinberg RA: Loss of E-cadherin promotes metastasis via multiple downstream transcriptional pathways. Cancer Res 2008, 68:3645-3654

20. Derksen, .W.B., Liu X, Saridin F, van der Guifden H, Zevenhoven J, Evers B, von Beijnum JR, Griffioen AW, Vink J, Krimpenfont P, Peterse $\mathrm{J}$, Cardiff R, Berns A, and Jonkers J: Somatic inactivation of Ecadherin and p53 in mice leads to metastatic lobular mammary carcinoma through induction of anoikis resistance and angiogenesis. Cancer Cell 2006, 10:437-449

21. Frisch SM, Vuori K, Ruoslathi E, Chan-Hui PY: Control of adhesiondependent cell survival by focal adhesion kinase. J Cell Biol 1996, 134:793-799

22. Demers MJ, Thibodeau S, Noel D, Fujita N, Tsuruo T, Gauthier R, Arguin $\mathrm{M}$, Vachon $\mathrm{PH}$ : Intestinal epithelial cancer cell anoikis resistance: eGFR-mediated sustained activation of Src overrides Fakdependent signaling to MEK/Erk and/or PI3-K/Akt-1. J Cell Biochem 2009, 107:639-654

23. Shain KH, Landowski TH, Dalton WS: Adhesion-mediated intracellular redistribution of $\mathrm{C}$-Fas-associated death domain-like IL-1-converting enzyme-like inhibitory protein-long confers resistance to CD95-induced apoptosis in hematopoietic cancer cell lines. J Immunol 2002, 168:2544-2553

24. Orford K, Orford CC, Byers SW: Exogenous expression of $\beta$-catenin regulates contact inhibition, anchorage-independent growth, anoikis, and radiation-induced cell cycle arrest. J Cell Biol 1999, 146:855-868

25. Laguigne LM, Samara RN, Wang W, El-Deiry WS, Corner G, Augenlicht L, Mishra L, Jessup JM: DR5 receptor mediates anoikis in human colorectal carcinoma cell lines Cancer Res 2008, 68:909-917

26. Scaffidi C, Schmitz I, Krammer PH, Peter ME: The role of C-FLIP in modulation of CD95-induced apoptosis. J Biol Chem 1999, 274: 1541-1548

27. Aoudjit F, Vuori K: Matrix attachment regulates Fas-induced apoptosis in endothelial cells: a role for c-flip and implications for anoikis. J Cell Biol 2001, 152:633-643

28. Simpson CD, Anyiwe K, Schimmer AD: Anoikis resistance and tumor metastasis. Cancer Lett 2008, 272:177-185

29. Mawji IA, Simpson CD, Gronda M, Williams MA, Hurren R, Henderson CJ, Datti A, Wrana JL, Schimmer AD: A chemical screen identifies anisomycin as an anoikis sensitizer that functions by decreasing FLIP protein synthesis. Cancer Res 2007, 67:8307-8315

30. Kurenova E, Xu, L-H, Yang X, Baldwin AS Jr, Craven RJ, Hanks SK, Liu, ZG, Cance WG: Focal adhesion kinase suppresses apoptosis by binding to the death domain of receptor-interacting protein. Mol Cell Biol 2004, 24:4361-4371

31. Kamajaran P, Burnek J, Nunez G, Kaplla YL: Receptor-interacting 
protein shuttles between cell death and survival signaling pathways. Mol Biol Cell 2010, 3:481-488

32. Tsuji T, Ibaragi S, Hu GF: Epithelial-mesenchymal transition and cell cooperativity in metastasis. Cancer Res 2009, 69:7135-7139

33. Zhu ML, Kyprianou N: Role of androgens and the androgen receptor in epithelial-mesenchymal transition and invasion of prostate cancer cells. FASEB J 2010, 24:769-777

34. Rohwer N, Welzel M, Daskalow K, Pfander D, Wiedenmann B, Detjen $\mathrm{K}$, Cramer T: Hypoxia-inducible factor $1 \alpha$ mediates anoikis resistance via suppression of $\alpha 5$ integrin. Cancer Res 2008, 68:10113-10120

35. Lee GY, Kenny PA, Lee EH, Bissell MJ: Three-dimensional culture models of normal and malignant breast epithelial cells. Nat Methods 2007, 4:359-365

36. Fournier MV, Fata JE, Martin KJ, Yaswen P, Bissell MJ: Interaction of E-cadherin and PTEN regulates morphogenesis and growth arrest in human mammary epithelial cells. Cancer Res 2009, 69:4545-4552

37. Fung C, Lock R, Gao S, Salas E, Debnath J: Induction of autophagy during extracellular matrix detachment promotes cell survival. Mol Biol Cell 2008, 19:797-806

38. Mills KR, Reginato M, Debnath J, Queenan B, Brugge JS: Tumor necrosis factor-related apoptosis-inducing ligand (TRAIL) is required for induction of autophagy during lumen formation in vitro. Proc Natl Acad Sci USA 2004, 101:3438-3443

39. Debnath J, Baehrecke EH, Kroemer G: Does autophagy contribute to cell death?. Autophagy 2005, 1:66-74

40. Sinha S, Levine B: The autophagy effector Beclin 1: a novel BH3-only protein, Oncogene 2008, 27(Suppl 1):S137-S148

41. Pattingre S, Tassa A, Qu X, Garuti R, Liang XH, Mizushima N, Packer M, Schneider MD, Levine B: Bcl-2 antiapoptotic proteins inhibit Beclin 1-dependent autophagy. Cell 2005, 122:927-939

42. Maiuri MC, Criollo A, Tasdemir E, Vicencio JM, Tajeddine N, Hickman JA, Geneste O, Kroemer G: BH3-only proteins and $\mathrm{BH} 3$ mimetics induce autophagy by competitively disrupting the interaction between Beclin 1 and $\mathrm{Bcl}-2 / \mathrm{Bcl}-\mathrm{X}_{\mathrm{L}}$ ). Autophagy 2007, 3:374-376

43. Luo S, Rubinsztein DC: Apoptosis blocks Beclin 1-dependent autophagosome synthesis: an effect rescued by Bcl-XL. Cell Death Differ 2010, 17:268-277

44. Li AE, Ito H, Rovira II, Kim KS, Takeda K, Yu ZY, Ferrans VJ, Finkel T: A role for reactive oxygen species in endothelial cell anoikis. Circ Res 1999, 85:304-310

45. Giannoni E, Buricchi F, Grimaldi G, Parri M, Cialdai F, Taddei ML, Raugei G, Ramponi G, Chiarugi P: Redox regulation of anoikis: reactive oxygen species as essential mediators of cell survival. Cell Death Differ 2008, 15:867-878

46. Giannoni E, Fiaschi T, Ramponi G, Chiarugi P: Redox regulation of anoikis resistance of metastatic prostate cancer cells: key role for Src and EGFR-mediated pro-survival signals. Oncogene 2009, 28:2074-2086

47. Edick MJ, Tesfay L, Lamb LE, Knudsen BS, Miranti CK: Inhibition of integrin-mediated crosstalk with epidermal growth factor receptor/Erk or Src signaling pathways in autophagic prostate epithelial cells induces caspase-independent death. Mol Biol Cell 2007, 18:2481-2490

48. Schafer ZT, Grassian AR, Song L, Jiang Z, Gerhart-Hines Z, Irie HY, Gao S, Puigserver P, Brugge JS: Antioxidant and oncogene rescue of metabolic defects caused by loss of matrix attachment. Nature 2009, 461:109-113

49. Reginato MJ, Mills KR, Paulus JK, Lynch DK, Sgroi DC, Debnath J, Muthuswamy SK, Brugge JS: Integrins and EGFR coordinately regulate the pro-apoptotic protein Bim to prevent anoikis. Nat Cell Biol 2003, 5:733-740

50. Wang X, Lin G, Martins-Taylor K, Zeng H, Xu RH: Inhibition of caspase-mediated anoikis is critical for bFGF-sustained culture of human pluripotent stem cells. J Biol Chem 2009, 284:34054-34064

51. Neiva KG, Zhang Z, Miyazawa M, Warner KA, Karl E, Nor JE: Cross talk initiated by endothelial cells enhances migration and inhibits anoikis of squamous cell carcinoma cells through STAT3/Akt/ERK signaling. Neoplasia 2009, 11:583-593

52. Rul W, Zugasti O, Roux P, Peyssonnaux C, Eychene A, Franke TF, Lenormand P, Fort P, Hibner U: Activation of ERK, controlled by Rac1 and Cdc42 via Akt, is required for anoikis. Ann NY Acad Sci 2002, 973:145-148

53. Ma L, Chen Z, Erdjument-Bromage H, Tempst P, Pandolfi PP: Phosphorylation and functional inactivation of TSC2 by Erk implications for tuberous sclerosis and cancer pathogenesis. Cell 2005, 121:179-193
54. Roux PP, Ballif BA, Anjum R, Gygi SP, Blenis J: Tumor-promoting phorbol esters and activated Ras inactivate the tuberous sclerosis tumor suppressor complex via p90 ribosomal S6 kinase. Proc Natl Acad Sci USA 2004, 101:13489-13494

55. Wang J, Whiteman MW, Lian H, Wang G, Singh A, Huang D, Denmark $\mathrm{T}$ : A non-canonical MEK/ERK signaling pathway regulates autophagy via regulating Beclin 1. J Biol Chem 2009, 284:21412-21424

56. Horbinski C, Chu CT: Kinase signaling cascades in the mitochondrion: a matter of life or death. Free Radic Biol Med 2005, 38:2-11

57. Kulich SM, Chu CT: Sustained extracellular signal-regulated kinase activation by 6-hydroxydopamine: implications for Parkinson's disease. J Neurochem 2001, 77:1058-1066

58. Kulich SM, Horbinski C, Patel M, Chu CT: 6-Hydroxydopamine induces mitochondrial ERK activation. Free Radic Biol Med 2007, 43:372-383

59. Cheng H, Liu P, Wang ZC, Zou L, Santiago S, Garbitt V, Gjoerup OV Iglehart JD, Miron A, Richardson AL, Hahn WC, and Zhao JJ: SIK1 couples LKB1 to p53-depedent anoikis and suppresses metastasis. Sci Signal 2009, 2:35-40

60. Zhang Y, Lu H, Dazin P, Kapila Y: Squamous cell carcinoma cell aggregates escape suspension-induced, p53-mediated anoikis: fibronectin and integrin $\alpha \vee$ mediate survival signals through focal adhesion kinase. J Biol Chem 2004, 279:48342-48349

61. Feng Z, Zhang H, Levine AJ, Jin S: The coordinate regulation of the p53 and mTOR pathways in cells. Proc Natl Acad Sci USA 2005, 102:8204-8209

62. Tasdemir E, Maiuri MC, Galluzzi L, Vitale I, Djavaheri-Mergny M, D'Amelio M, Criollo A, Morselli E, Zhu C, Harper F, Nannmark U, Samara C, Pinton P, Vicencio JM, Carnuccio R, Moll UM, Madeo F, Paterlini-Brechot P, Rizzuto R, Szabadkai G, Pierron G, Blomgren K, Tavernarakis N, Codogno P, Cecconi F, Kroemer G: Regulation of autophagy by cytoplasmic p53. Nat Cell Biol 2008, 10:676-687

63. Bialik S, Kimchi A: The death-associated protein kinases: structure, function, and beyond. Annu Rev Biochem 2006, 75:189-210

64. Chen CH, Wang WJ, Kuo JC, Tsai HC, Lin JR, Chang ZF, Chen RH: Bidirectional signals transduced by DAPK-ERK interaction promote the apoptotic effect of DAPK. EMBO J 2005, 24:294-304

65. Wang WJ, Kuo JC, Yao CC, Chen RH: DAP-kinase induces apoptosis by suppressing integrin activity and disrupting matrix survival signals. J Cell Biol 2002, 159:169-179

66. Gozuacik D, Bialik S, Raveh T, Mitou G, Shohat G, Sabanay H, Mizushima N, Yoshimori T, Kimchi A: DAP-kinase is a mediator of endoplasmic reticulum stress-induced caspase activation and autophagic cell death. Cell Death Differ 2008, 15:1875-1886

67. Inbal B, Bialik S, Sabanay I, Shani G, Kimchi A: DAP kinase and DRP-1 mediate membrane blebbing and the formation of autophagic vesicles during programmed cell death. J Cell Biol 2002, 157:455-468

68. Zalckvar E, Berissi H, Eisenstein M, Kimchi A: Phosphorylation of Beclin 1 by DAP-kinase promotes autophagy by weakening its interactions with Bcl-2 and Bcl-XL. Autophagy 2009, 5:720-722

69. Zalckvar E, Berissi H, Mizrachy L, Idelchuk Y, Koren I, Eisenstein M, Sabanay H, Pinkas-Kramarski R, Kimchi A: DAP-kinase-mediated phosphorylation on the $\mathrm{BH} 3$ domain of beclin 1 promotes dissociation of beclin 1 from Bcl-XL and induction of autophagy. EMBO Rep 2009, 10:285-292

70. Stevens C, Lin Y, Harrison B, Burch L, Ridgway RA, Sansom O, Hupp T: Peptide combinatorial libraries identify TSC2 as a death-associated protein kinase (DAPK) death domain-binding protein and reveal a stimulatory role for DAPK in mTORC1 signaling. J Biol Chem 2009 284:334-344

71. Li H, Ray G, Yoo BH, Erdogan M, Rosen KV: Down-regulation of death-associated protein kinase-2 is required for $\beta$-catenin-induced anoikis resistance of malignant epithelial cells. J Biol Chem 2009, 284:2012-2022

72. Sasnauskiene A, Kadziauskas J, Vezelyte N, Jonusiene V, Kirveliene $\mathrm{V}$ : Apoptosis, autophagy and cell cycle arrest following photodamage to mitochondrial interior. Apoptosis 2009, 14:276-286

73. Rodríguez-Hernández A, Cordero MD, Salviati L, Artuch R, Pineda M, Briones P, Gomez Izquierdo L, Cotan D, Navas P, Sanchez-Alcazar JA: Coenzyme $Q$ deficiency triggers mitochondria degradation by mitophagy. Autophagy 2009, 5:19-32

74. Liang XH, Jackson S, Seaman M, Brown K, Kempkes B, Hibshoosh H, Levine $\mathrm{B}$ : Induction of autophagy and inhibition of tumorigenesis by beclin 1. Nature 1999, 402:672-676 
75. Qu X, Yu J, Bhagat G, Furuya N, Hibshoosh H, Troxel A, Rosen J, Eskelinen EL, Mizushima N, Ohsumi Y, Cattoretti G, Levine B: Promotion of tumorigenesis by heterozygous disruption of the beclin 1 autophagy gene. J Clin Invest 2003, 112:1809-1820

76. Yue Z, Jin S, Yang C, Levine AJ, Heintz N: Beclin 1, an autophagy gene essential for early embryonic development, is a haploinsufficient tumor suppressor. Proc Natl Acad Sci USA 2003, 100: 15077-15082

77. Mathew R, Karp CM, Beaudoin B, Vuong N, Chen G, Chen HY, Bray K, Reddy A, Bhanot G, Gelinas C, Dipaola RS, Karantza-Wadsworth $V$, White E: Autophagy suppresses tumorigenesis through elimination of p62. Cell 2009, 137:1062-1075

78. Mathew R, Kongara S, Beaudoin B, Karp CM, Bray K, Degenhardt K, Chen G, Jin S, White E: Autophagy suppresses tumor progression by limiting chromosomal instability. Genes Dev 2007, 21:1367-1381

79. Moscat J, Diaz-Meco MT: p62 at the crossroads of autophagy, apoptosis, and cancer. Cell 2009, 137:1001-1004

80. Yoo BH, Wu X, Li Y, Haniff M, Sasazuki T, Shirasawa S, Eskelinen EL, Rosen KV: Oncogenic ras-induced downregulation of autophagy mediator Beclin-1 is required for malignant transformation of intestinal epithelial cells. J Biol Chem 2010, 285:5438-5449

81. Carew JS, Medina EC, Esquivel JA 2nd, Mahalingam D, Swords R, Kelly K, Zhang H, Huang P, Mita AC, Mita MM, Giles FJ, Nawrocki ST: Autophagy inhibition enhances vorinostat-induced apoptosis via ubiquitinated protein accumulation. J Cell Mol Med 2009, doi: 10.1111/j.1582-4934.2009.00832.x

82. Bijnsdorp IV, Peters GJ, Temmink OH, Fukushima M, Kruyt FA: Differential activation of cell death and autophagy results in an increased cytotoxic potential for trifluorothymidine compared to 5 -fluorouracil in colon cancer cells. Int J Cancer 2010, 126:2457-2468

83. Lomonaco SL, Finniss S, Xiang C, Decarvalho A, Umansky F, Kalkanis $\mathrm{SN}$, Mikkelsen T, Brodie C: The induction of autophagy by gammaradiation contributes to the radioresistance of glioma stem cells. Int $J$ Cancer 2009, 125:717-722

84. Yoo BH, Wu X, Derouet M, Haniff M, Eskelinen EL, Rosen K: Hypoxiainduced downregulation of autophagy mediator beclin 1 reduces the susceptibility of malignant intestinal epithelial cells to hypoxia-dependent apoptosis. Autophagy 2009, 5:1166-1179

85. Kiyono K, Suzuki HI, Matsuyama H, Morishita Y, Komuro A, Kano MR, Sugimoto K, Miyazono K: Autophagy Is activated by TGF- $\beta$ and potentiates TGF- $\beta$-mediated growth inhibition in human hepatocellular carcinoma cells. Cancer Res 2009, 69:8844-8852

86. Sun $\mathrm{Y}$, Zhang J, Peng ZL: Beclin1 induces autophagy and its potential contributions to sensitizes $\mathrm{SiHa}$ cells to carboplatin therapy. Int $J$ Gynecol Cancer 2009, 19:772-776

87. Sakamoto S, McCann RO, Dhir, R. and Kyprianou N: Talin1 promotes tumor invasion and metastasis via focal adhesion signaling and anoikis resistance. Cancer Res 2010, 70:1885-1895 